\title{
Shear-induced particle diffusion and longitudinal velocity fluctuations in a granular-flow mixing layer
}

\author{
By S. S. HSIAU AND M. L. HUNT \\ Division of Engineering and Applied Science, California Institute of Technology, Pasadena, \\ CA 91125 , USA
}

(Received 14 November 1991 and in revised form 8 November 1992)

In flows of granular material, collisions between individual particles result in the movement of particles in directions transverse to the bulk motion. If the particles were distinguishable, a macroscopic overview of the transverse motions of the particles would resemble a self-diffusion of molecules as occurs in a gas. The present granularflow study includes measurements of the self-diffusion process, and of the corresponding profiles of the average velocity and of the streamwise component of the fluctuating velocity. The experimental facility consists of a vertical channel fed by an entrance hopper that is divided by a splitter plate. Using differently-coloured but otherwise identical glass spheres to visualize the diffusion process, the flow resembles a classic mixing-layer experiment. Unlike molecular motions, the local particle movements result from shearing of the flow; hence, the diffusion experiments were performed for different shear rates by changing the sidewall conditions of the test section, and by varying the flow rate and the channel width. In addition, experiments were also conducted using different sizes of glass beads to examine the scaling of the diffusion process. A simple analysis based on the diffusion equation shows that the thickness of the mixing layer increases with the square-root of downstream distance and depends on the magnitude of the velocity fluctuations relative to the mean velocity. The results are also consistent with other studies that suggest that the diffusion coefficient is proportional to the particle diameter and the square-root of the granular temperature.

\section{Introduction}

In recent years the field of granular material flows has significantly progressed through the application of gas-kinetic theories (Savage \& Jeffrey 1981; Jenkins \& Savage 1983; Lun et al. 1984), and by advances in the computer simulations of the flows (Campbell \& Brennen 1985; Walton \& Braun 1986; Campbell 1989). For these studies, an analogy is drawn between the movement of molecules or atoms due to the thermodynamic temperature and the velocity fluctuations due to shearing of the material. In this context, the term granular temperature is used to quantify the square of the fluctuating velocities of the granular material. Unlike molecular velocities which depend on the square-root of the gas thermodynamic temperature, the granular temperature is generated through the particle collisions and through the random movements of the particles (Campbell 1990). Besides the kinetic-theory and the molecular-dynamics studies, several investigations have emphasized experimental measurements that include the distributions in the local velocity and the density. Two recent studies, Johnson, Nott \& Jackson (1990) and Ahn, Brennen \& Sabersky (1991), investigated flows down an inclined chute, and used fibre optic probes for the velocity 
measurements. The study by Ahn et al. also included measurements of the fluctuating velocity in the longitudinal direction.

In addition to the work that focuses on the momentum transport relations, several studies have focused on problems involving other transport processes such as particle diffusion. In granular flows, the spatial velocity variations as quantified by the granular temperature result in a mixing or diffusion of the particles that is similar to molecular diffusion in gases. The kinetic-theory based studies by Savage (1992) and by Hsiau \& Hunt (1992) develop an expression for the particle diffusion coefficient that is proportional to the product of the square-root of the granular temperature and the particle diameter. This functional dependence of the diffusion coefficient follows from the results of gas kinetic theories in which the gas self-diffusion coefficient depends directly on the square-root of the thermodynamic temperature and inversely on the product of the number density and the scattering cross-section (Kennard 1938; Chapman \& Cowling 1971). In granular flow terms, the corresponding self-diffusion coefficient would depend on the particle diameter and the square-root of the granular temperature. The granular temperature would then depend on the shear rate of the flow. A similar dependence of the diffusion process on the flow shear rate is noted in several experimental studies, including the work by Hwang \& Hogg (1980), Bridgwater (1980) and Buggisch \& Loeffelmann (1989). Hwang \& Hogg examined particle mixing in an inclined chute; Bridgwater investigated diffusion that resulted during plane shearing of a layer of granular material, and Buggisch \& Loffelmann experimented with a two-dimensional shearing of differently coloured cylindrical rods. These studies did not include measurements of the local velocity distribution or of the velocity fluctuations.

In heat transfer problems involving flows of granular materials, the local movements of the particles result in an increase in thermal transport. Wang \& Campbell (1992) used a Couette flow apparatus with an imposed temperature gradient to measure the overall heat flux. From the experimental results they determined that the effective thermal conductivity of the material increased linearly with the shear rate. Since an increase in shear rate yields an increase in granular temperature, the experiments indicate that the thermal transport depends on the movement of the particles. The kinetic-theory analysis by Hsiau \& Hunt (1992) also shows that the effective thermal conductivity of a granular flow increases with the square-root of the granular temperature.

The present work was motivated by an interest in examining diffusion in granular material flows as would be important in mixing studies or in heat transfer studies. The experiment resembled a classic mixing-layer flow using a gravity-fed vertical channel and differently-coloured but otherwise identical particles. The flow shear rate was changed by varying the channel width, the surface conditions of the channel walls, and the flow rate. The velocity measuring technique developed by Ahn (1989) and reported by Ahn et al. (1991) was used to determine the longitudinal ensemble-average velocity and the square of the velocity fluctuations. The diffusion process was examined using an image processing system and measuring the growth of the mixing-layer thickness with downstream position. A preliminary report of the first experimental results is found in Hunt \& Hsiau (1992).

\section{Experimental apparatus and instrumentation}

The granular-flow mixing-layer facility is shown in figure 1 . The test section is $1 \mathrm{~m}$ in height, $2.18 \mathrm{~cm}$ in depth, and has adjustable sidewalls of half-width $H$. An upper 


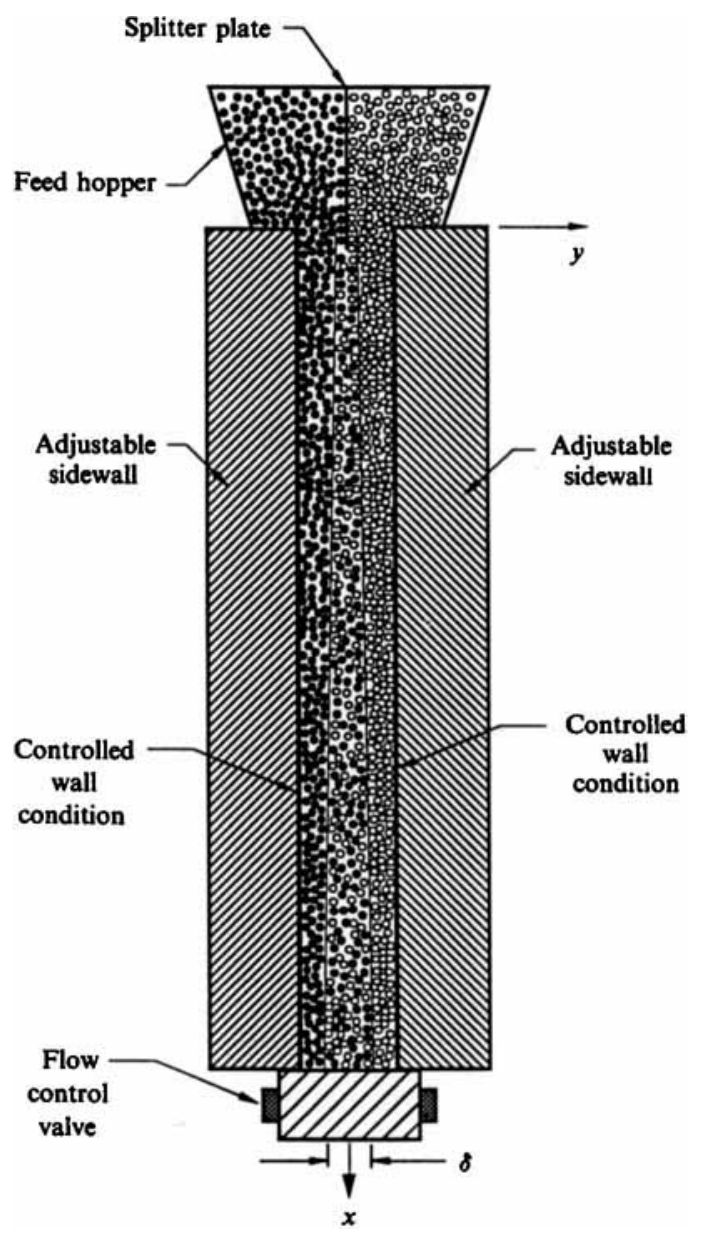

FIGURE 1. Experimental mixing-layer facility.

hopper feeds the particles into the test section. A thin metal splitter plate divides the hopper and extends approximately $2 \mathrm{~cm}$ into the test section. On one side of the splitter plate the particles have been dyed black, and on the other side the particles are a fluorescent yellow; aside from the difference in colour, the particles are identical glass spheres. The size of the opening of the lower hopper controls the material flow rate. The flow rate is determined by collecting the material exiting the test section over a designated period of time and weighing the sample.

By using polished tempered-glass plates for the front and the back surfaces, the particles slipped past the surface with minimal shearing, creating a two-dimensional flow. To minimize the effects of dirt and static electric charge, the front and back glass plates were removed after two to three experimental runs to be cleaned and polished. As shown by the experimental results, the surface conditions on the sidewalls influenced the velocity profiles and the diffusion experiments. The present experiments comprise three sidewall conditions: polished glass; walls roughened by adhering a layer of $3 \mathrm{~mm}$ diameter glass spheres to the surface; and aluminium walls with $120^{\circ}$ sawtooth V-grooves of $3 \mathrm{~mm}$ depth.

Using a technique developed by Ahn (1989) the velocity in the flow direction was measured using two $1.6 \mathrm{~mm}$ diameter fibre-optic displacement probes that were spaced 

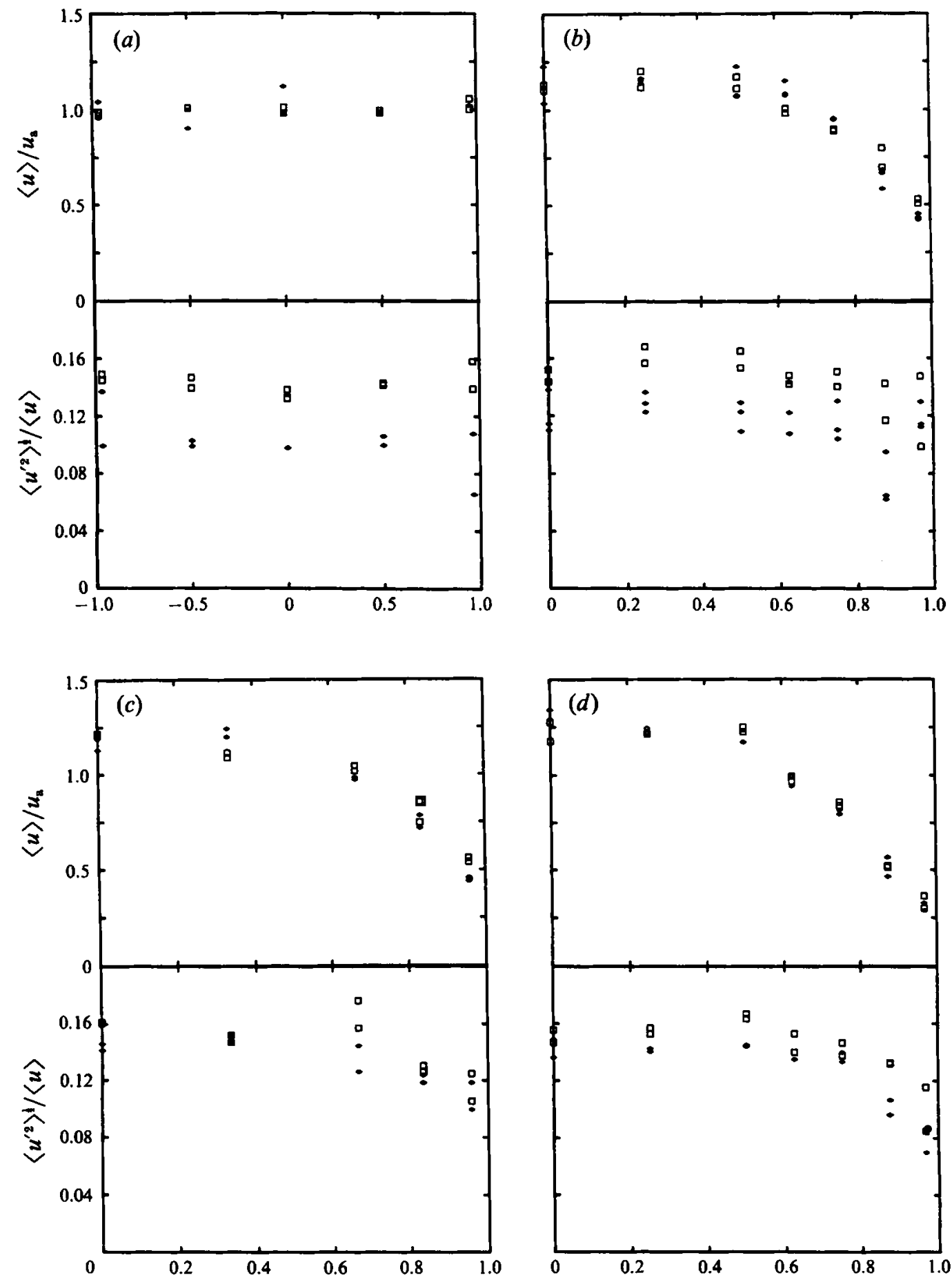

Channel location, $y / H$

Figure $2(a-d)$. For caption see facing page. 

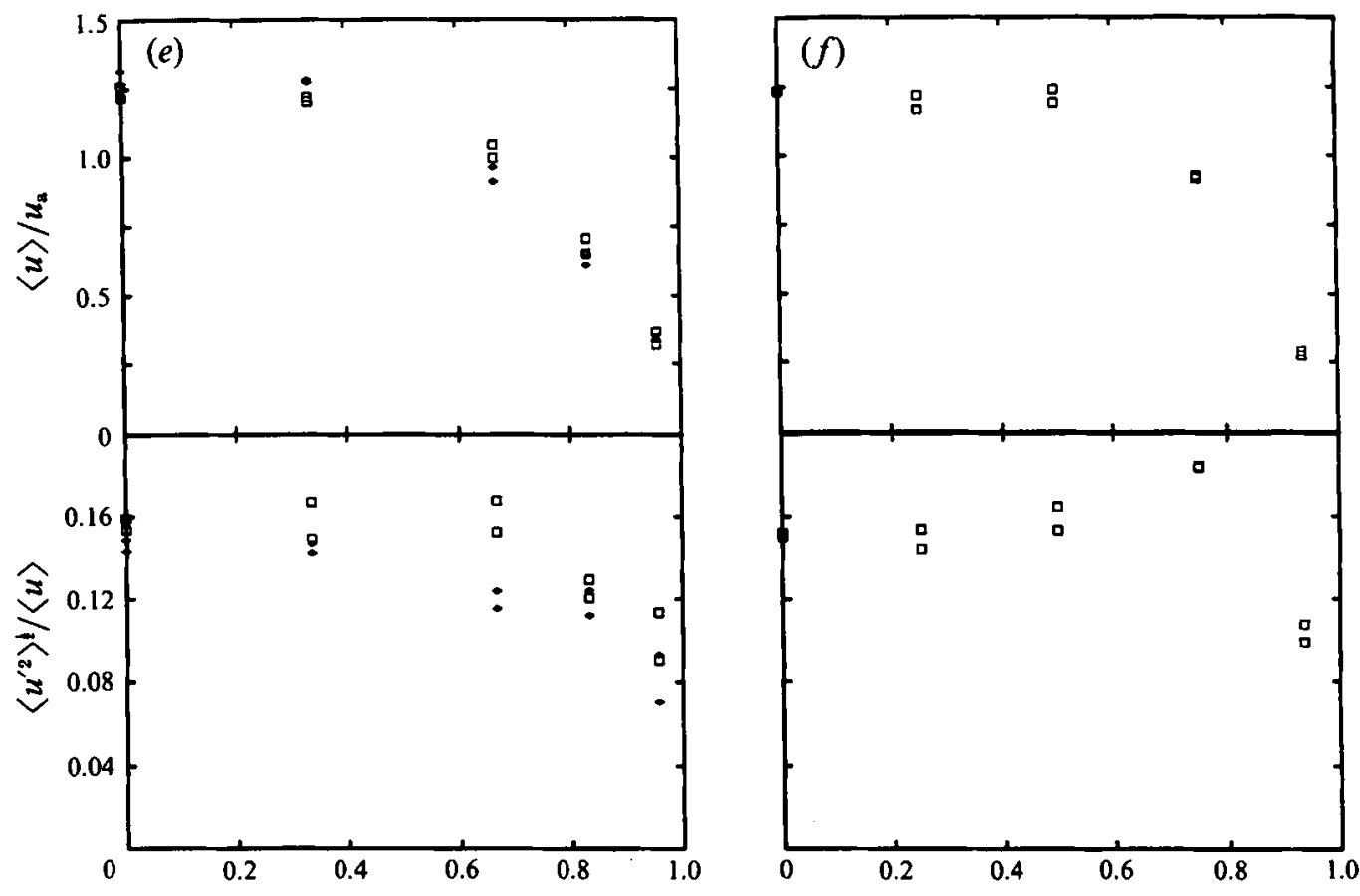

Channel location, $y / H$

FIGURE 2. (a-e) Local and fluctuating velocity distributions using $3 \mathrm{~mm}$ glass beads for: $(a)$ smoothwalled channel, $2 H=5.1 \mathrm{~cm}, u_{\mathrm{a}}=4.5 \mathrm{~cm} / \mathrm{s}$ for $\dot{m}=0.080 \mathrm{~kg} / \mathrm{s}$, and $u_{\mathrm{a}}=10.1 \mathrm{~cm} / \mathrm{s}$ for $\dot{m}=$ $0.18 \mathrm{~kg} / \mathrm{s} ;(b)$ rough-walled wide channel, $2 H=5.1 \mathrm{~cm}, u_{\mathrm{a}}=4.1 \mathrm{~cm} / \mathrm{s}$ for $\dot{m}=0.075 \mathrm{~kg} / \mathrm{s}$, and $u_{\mathrm{a}}=$ $10.3 \mathrm{~cm} / \mathrm{s}$ for $\dot{m}=0.19 \mathrm{~kg} / \mathrm{s} ;(c)$ rough-walled narrow channel, $2 H=3.8 \mathrm{~cm}, u_{\mathrm{a}}=5.4 \mathrm{~cm} / \mathrm{s}$ for $\dot{m}=$ $0.070 \mathrm{~kg} / \mathrm{s}$, and $u_{\mathrm{a}}=9.7 \mathrm{~cm} / \mathrm{s}$ for $\dot{m}=0.13 \mathrm{~kg} / \mathrm{s} ;(d)$ wide channel with sawtooth walls, $2 H=5.1 \mathrm{~cm}$, $u_{\mathrm{a}}=5.1 \mathrm{~cm} / \mathrm{s}$ for $\dot{m}=0.097 \mathrm{~kg} / \mathrm{s}$, and $u_{\mathrm{a}}=10.9 \mathrm{~cm} / \mathrm{s}$ for $\dot{m}=0.20 \mathrm{~kg} / \mathrm{s} ;(e)$ narrow channel with sawtooth walls, $2 H=3.8 \mathrm{~cm}, u_{\mathrm{a}}=4.9 \mathrm{~cm} / \mathrm{s}$ for $\dot{m}=0.071 \mathrm{~kg} / \mathrm{s}, u_{\mathrm{a}}=9.7 \mathrm{~cm} / \mathrm{s}$ for $\dot{m}=0.13 \mathrm{~kg} / \mathrm{s}: \square$, high flow rate; $\diamond$, low flow rate. $(f)$ Using $2 \mathrm{~mm}$ diameter beads with sawtooth walls, $2 H=2.54 \mathrm{~cm}$, $u_{\mathrm{a}}=18.5 \mathrm{~cm} / \mathrm{s}$ and $\dot{m}=0.15 \mathrm{~kg} / \mathrm{s}$.

centre-to-centre $4.5 \mathrm{~mm}$ apart for $3 \mathrm{~mm}$ beads, and $2.5 \mathrm{~mm}$ apart for the $2 \mathrm{~mm}$ beads. The local streamwise velocity was calculated from the passage time of a particle past the two probes and from the spacing between the probes. By averaging over 100-200 particles the ensemble-average velocity, $\langle u\rangle$, and the ensemble-average of the square of the fluctuating velocity, $\left\langle u^{\prime 2}\right\rangle$, were obtained for a specific channel location. This method of velocity measurement was calibrated using a rotating wheel with a layer of particles attached to the outer periphery that revolved at a fixed speed; the calibration indicated a $2-3 \%$ error in the average velocity measurements.

The diffusion experiments involved the visual observation of the coloured particles through the front glass plate. The flow was filmed using a commercial video recorder, and the images were digitized in real time using a frame-grabber board that was operated by a personal computer. To increase the number of pixels per particle (approximately 3-4 pixels are used for a distance of one particle diameter), the upper and lower halves of the channel were filmed separately. A digitized image was recorded at a rate of 3 images per s for a total of 50 to 90 images. The buffer size of the framegrabber board limited the total number of images that could be stored. After recording the digitized images, the contrast between the light and dark particles was enhanced so that the dark particles appear black and the light particles appear white. The local ensemble-average colour concentration was calculated from the enhanced images. 
From the averaged images, the thickness of the mixing layer, $\delta$, was determined from the transverse distance over which the colour varied from 0.01 to 0.99 where 0 indicates black and 1.0 indicates white. This image-processing method was tested on known distributions of particles. These tests indicated that the results were affected by the lighting and by non-uniform colouring of the particles; in the actual experiments, care was taken to minimize these effects. After each experiment, the coloured beads were washed and dyed for the next experiment.

Most of the experiments were conducted using glass beads with an average diameter, $d$, of $3 \mathrm{~mm}$. For these experiments there were two different channel widths: a narrow channel of $3.8 \mathrm{~cm}(2 \mathrm{H} / \mathrm{d}=12.7)$, and a wide channel of $5.1 \mathrm{~cm}(2 \mathrm{H} / \mathrm{d}=17.0)$. For the roughened and sawtooth sidewalls, this distance was measured between the outer edges of the attached particles or between the tips of the peaks. The experiments have been performed for low and high flow rates, which correspond to average velocities of about 5 and $10 \mathrm{~cm} / \mathrm{s}$. Since the roughened and sawtooth walls offered a slightly larger flow cross-section, the mass flow rates were larger for these wall conditions. Some additional experiments were performed at higher flow rates; however, based on visual observations the flow appeared to surge, especially for the smooth and the roughened sidewalls.

For the roughened and sawtooth walls, a total of eight experiments were conducted using the $3 \mathrm{~mm}$ diameter beads for the two flow rates, and the two channel sizes. As shown in $\S 4$, the largest mixing-layer thickness was approximately $4 d$ at a downstream distance of $x / d=300$. To increase the mixing-layer thickness relative to the particle size, additional experiments were conducted using smaller beads of 1 and $2 \mathrm{~mm}$ diameter. For the $2 \mathrm{~mm}$ beads, the channel width using the sawtooth sides was $2.54 \mathrm{~cm}$, which corresponded to $2 \mathrm{H} / \mathrm{d}=12.7$ - the same ratio of width to particle diameter as in the narrow channel with the $3 \mathrm{~mm}$ beads. For the $1 \mathrm{~mm}$ beads, the smallest channel size possible was $1.59 \mathrm{~cm}(2 \mathrm{H} / d=15.9)$. With the smaller particle diameters, it was possible to use higher average velocities without an apparent surging problem. As a result, the average velocities using the 1 and $2 \mathrm{~mm}$ beads were approximately $18 \mathrm{~cm} / \mathrm{s}$. The velocity profiles were not measured for the $1 \mathrm{~mm}$ beads, because the uncertainty in the measurement technique increased significantly when the fibre optic probe (1.6 $\mathrm{mm}$ diameter) was larger than the size of the bead.

The average solid fraction, $v$, was determined from the equation $v=\dot{m} / \rho_{\mathrm{p}} u_{\mathrm{a}} A_{\mathrm{c}}$, where $\dot{m}$ is the mass flow rate, $\rho_{\mathrm{p}}$ is the particle density $\left(\rho_{\mathrm{p}}=2490 \mathrm{~kg} / \mathrm{m}^{3}\right), u_{\mathrm{a}}$ is the average velocity that is determined from the velocity profiles, and $A_{\mathrm{c}}$ is the channel cross-sectional area. Because of the additional flow area caused by the surfaces of the roughened and sawtooth channels, the effective channel width was estimated as the distance $2 H+d$. For all experiments, the solid fraction ranged from approximately 0.59 to 0.65 . The distribution of the local solid fraction was not measured, because the variation appeared to be small and within the uncertainty of the measurement technique.

\section{Average and fluctuating longitudinal velocity profiles}

In preliminary experiments, the velocity of the flow was measured at several locations along the channel to determine the distance over which the profile developed. The measurements showed that the velocity variation extended over an axial distance of approximately 3 to 4 particles, which was less than the distance that the splitter plate extended into the channel. The splitter plate did not cause a detectable variation in the velocity profiles. After this initial entrance region, no significant variation was detected 
with downstream position except in the region within $10 \mathrm{~cm}$ of the exit hopper. All of the following velocity measurements were obtained at a position approximately halfway down the length of the channel $(x=50 \mathrm{~cm})$ and are shown in terms of nondimensional distance $y / H$ measured from the channel centreline.

Figure 2 $(a-e)$ indicates the distribution in the ensemble-average local velocity for the $3 \mathrm{~mm}$ diameter spheres for five different channel conditions: the wide channel with glass sidewalls; the wide and narrow channels for the sphere-roughened walls; and the wide and narrow channels for the sawtooth walls. For the polished-glass sidewalls the velocity profile is relatively flat. For the roughened and sawtooth walls, the velocity profiles are flat in the central region, but show a significant variation near the sidewalls over a distance of roughly 3-3.5 particle diameters. The central plug-flow region appears to be proportionally smaller in the narrow channel. Similar profiles have been previously observed in vertical channel flows by Savage (1979). The data closest to the channel wall are taken $0.8 \mathrm{~mm}$ from the wall, which is the position closest to the wall that the centre of the $1.6 \mathrm{~mm}$ diameter fibre-optic probe can be placed. The velocity measured at this position is referred to as the slip velocity. The slip velocities in roughwalled channels are approximately $45-60 \%$ of the average velocity, and are from $25-40 \%$ for the sawtooth walls. As a result, the flow in the sawtooth channel has a slightly higher centreline velocity than that in the roughened channel for the same average velocity. The results shown in figure $2(f)$ are for the $2 \mathrm{~mm}$ diameter beads in the sawtooth channel of width $2 H / d=12.7$. The non-dimensional profiles appear to be similar to that shown in figure $2(e)$ for the $3 \mathrm{~mm}$ beads in the narrow channel $(2 H / d=12.7)$.

Figure $2(a-f)$ also includes the distributions in the square-root of the ensembleaverage of the square of the fluctuating velocity in the flow direction normalized by the local velocity. The fluctuating components in the central region of the channel are approximately $14-16 \%$ of the local velocity for the high flow rates, and between $10-14 \%$ for the low flow rates. The magnitude of the fluctuating velocity as a percentage of the local velocity does not vary significantly across the channel, although for the roughened and sawtooth channel there appears to be a slight decrease near the walls. It is important to note that the measuring technique is designed for unidirectional flows. If the particle velocity has a tangential component, as visual observations suggest for the particles closest to the walls, the downstream fibre-optic probe does not detect the particle trajectory. As a result of the transverse motion, the measurement of the streamwise component of the fluctuating velocity may decrease. The fluctuating velocity component for the $2 \mathrm{~mm}$ diameter beads is similar to that measured using the larger beads as shown by comparing figures $2(e)$ and $2(f)$. The scatter in the velocity measurements may result from variations in the surface conditions of the front and back plates, and in the conditions of the particles from experiment to experiment.

\section{Measurements of the mixing-layer thickness}

Particle diffusion experiments were made using the $3 \mathrm{~mm}$ diameter beads for both low and high flow rates, and for the three different sidewall conditions; in addition, two experiments were also performed using 1 and $2 \mathrm{~mm}$ beads. The photographs in figure 3 show typical particle distributions at $45<x<90 \mathrm{~cm}$ for four different experimental cases. Figure $3(a)$ is from a flow with polished-glass sidewalls and shows no net diffusion of the particles. Visual observations indicate that the black particles remain on one side of the channel and the yellow particles on the other. The picture also suggests that the splitter plate does not have an observable effect on the mixing process. 
(a)

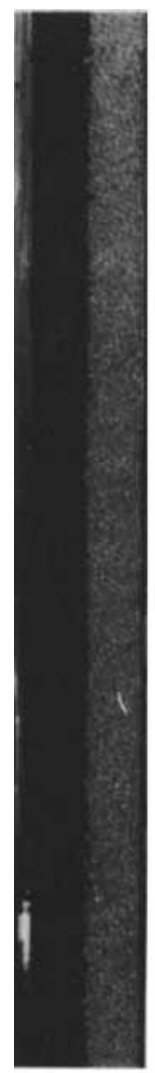

(b)

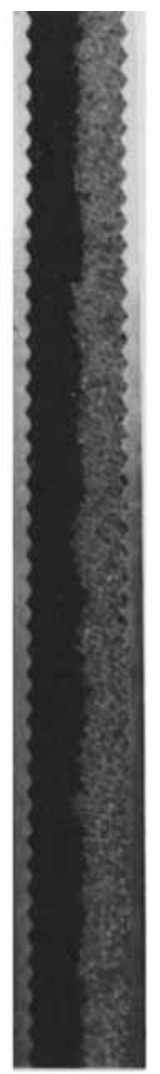

(c)

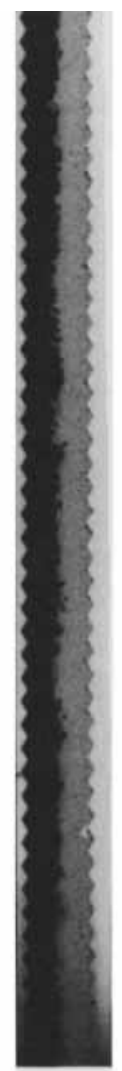

(d)

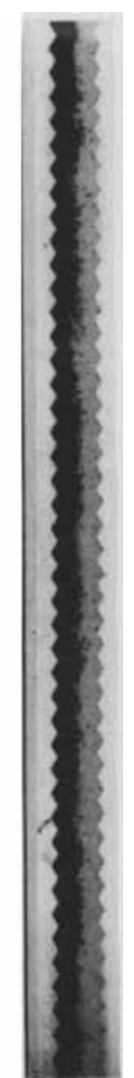

Figure 3. Typical particle distribution $(45<x<90 \mathrm{~cm})$ for $(a)$ the smooth-walled channel with $3 \mathrm{~mm}$ beads $(2 H=5.1 \mathrm{~cm}),(b)$ the sawtooth narrow channel with $3 \mathrm{~mm}$ beads $(2 H=3.8 \mathrm{~cm}),(c)$ the sawtooth channel with $2 \mathrm{~mm}$ beads $(2 H=2.54 \mathrm{~cm})$, and $(d)$ the sawtooth channel with $1 \mathrm{~mm}$ beads $(2 H=1.59 \mathrm{~cm})$.

When the flow is sheared, however, as occurs in the three other flows shown in figures $3(b), 3(c), 3(d)$, some particles move across the centreline. In figure $3(b)$, the particles are $3 \mathrm{~mm}$ diameter and the channel has the sawtooth walls. The interface between the dark and light particles is uneven, but few of the light particles appear to be surrounded by dark particles. Using the smaller beads, the diffusion process has increased because of the length of the channel as compared to the particle size is proportionally larger as shown in figures $3(c)$ and $3(d)$ for the 2 and $1 \mathrm{~mm}$ diameter beads. These pictures indicate that more of light particles have migrated into the region of the darker particles.

The diffusion results are shown in figures 4-7 for the glass-walled channel, for the rough-walled channels, for the sawtoothed channels, and for the 2 and $1 \mathrm{~mm}$ diameter beads, respectively. The figures indicate the thickness of the non-dimensional mixing layer, $\delta / d$, as a function of non-dimensional downstream distance, $x / d$. For glass sidewalls as shown in figure 4 , the mixing-layer thickness remains constant, close to one particle diameter in size. This result is anticipated from the photograph of figure 3(a), which indicates either a black or yellow particle lying at the centre of the channel. For the roughened and sawtoothed surfaces, the shearing of the flow results in some transverse movement of the particles. As indicated by figures $5-7$, the thicknesses of 


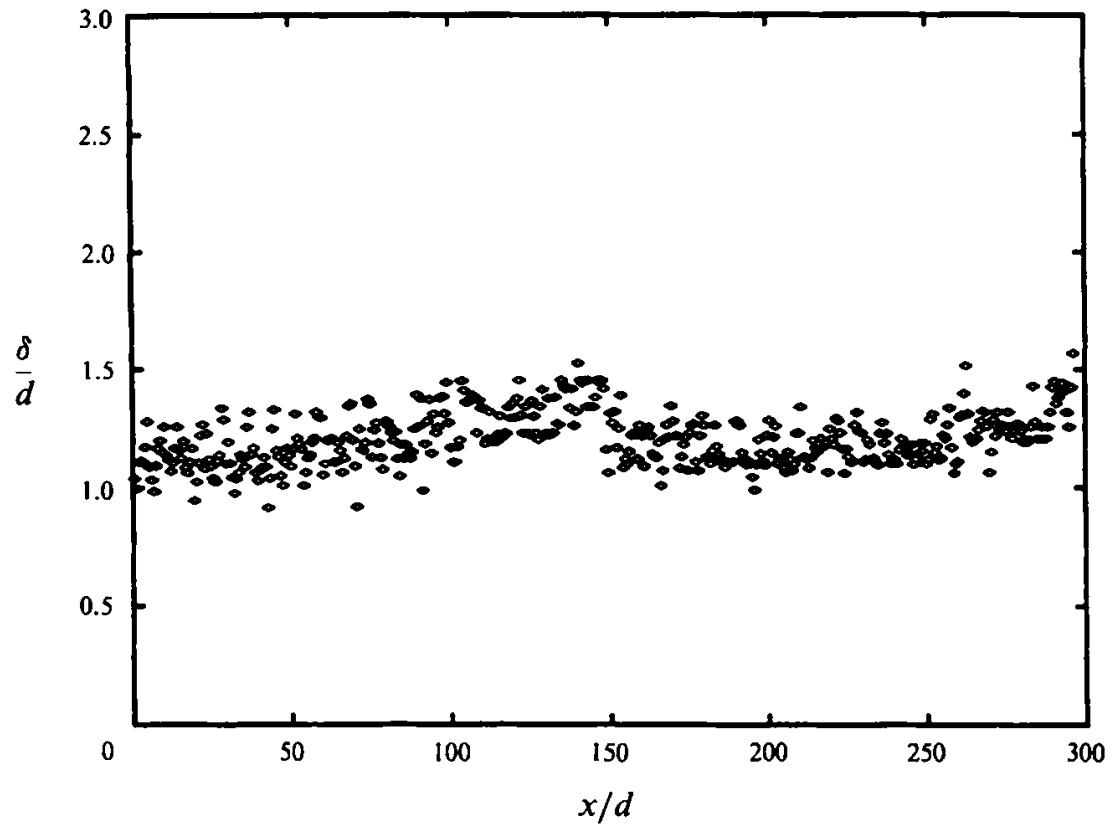

FIGURE 4. The growth of the mixing-layer thickness, $\delta / d$, as a function of downstream distance, $x / d$, for the smooth-walled channel, $\dot{m}=0.091 \mathrm{~kg} / \mathrm{s}$ and $2 H=5.1 \mathrm{~cm}$.

the mixing layers are greater than one particle diameter and increase with downstream position. The particle diffusion is more pronounced in the narrow channels; this result may be due to the proximity of the walls to the dark-particle/light-particle interface, which then increases the transverse particle motion within the centre of the channel. However, as indicated by the velocity measurements, the particle diffusion occurs in a region of the flow where there is no discernible longitudinal velocity gradient. In comparing the results in the roughened and sawtoothed channels, the diffusion in the sawtoothed channels appears to be slightly larger; the velocity profiles show that the velocity gradient at the walls is greater with the sawtooth sides and the larger shear rate may increase the transverse motion of the particles. By increasing the flow rate, the diffusion rates appear to increase slightly for all of the flows except for the wide roughened channel in which the mixing-layer thicknesses are comparable. With the larger non-dimensional channel length, the non-dimensional mixing-layer thickness at $x=90 \mathrm{~cm}$ is greater for the 2 and $1 \mathrm{~mm}$ beads, as shown in figures $7(a)$ and $7(b)$.

The curves in figures 5-7 are for flows of constant Péclet number, $P e=u_{\mathrm{m}} d / D_{\mathrm{m}}$, where $u_{\mathrm{m}}$ and $D_{\mathrm{m}}$ are the velocity and the diffusion coefficient. These curves are determined from a simple analysis of the flow based on the diffusion equation. Assuming that the flow is steady with no transverse velocity and neglecting diffusion in the flow direction, the diffusion equation for the mixing layer is written as

$$
u \frac{\partial C}{\partial x}=\frac{\partial}{\partial y}\left(D \frac{\partial C}{\partial y}\right)
$$

where $u$ is the local velocity, $C$ indicates the colour concentration, and $D$ is the local diffusion coefficient. If within the mixing layer the velocity and diffusion coefficient are assumed to be constant, the diffusion equation may be rewritten as

$$
u_{\mathrm{m}} \frac{\partial C}{\partial x}=D_{\mathrm{m}} \frac{\partial^{2} C}{\partial y^{2}}
$$



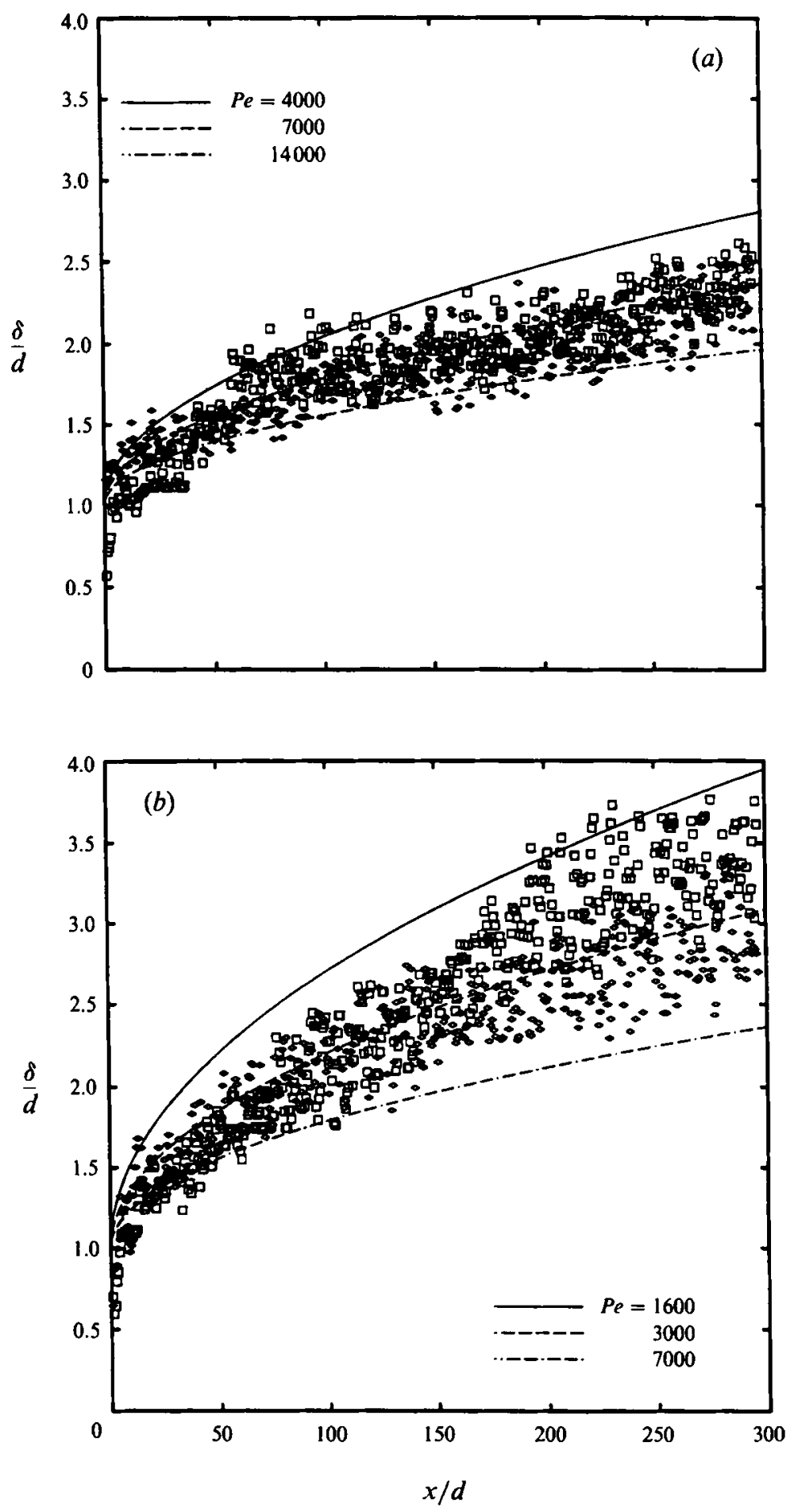

FIGURE 5. The growth of the mixing-layer thickness, $\delta / d$, as a function of downstream distance, $x / d$, for $(a)$ the wide rough-walled channel, low flow rate $\dot{m}=0.10 \mathrm{~kg} / \mathrm{s}(\diamond)$, and high flow rate $\dot{m}=$ $0.20 \mathrm{~kg} / \mathrm{s}(\square) ;(b)$ the narrow rough-walled channel, $\dot{m}=0.070 \mathrm{~kg} / \mathrm{s}(\diamond)$, and $\dot{m}=0.13 \mathrm{~kg} / \mathrm{s}(\square)$. 

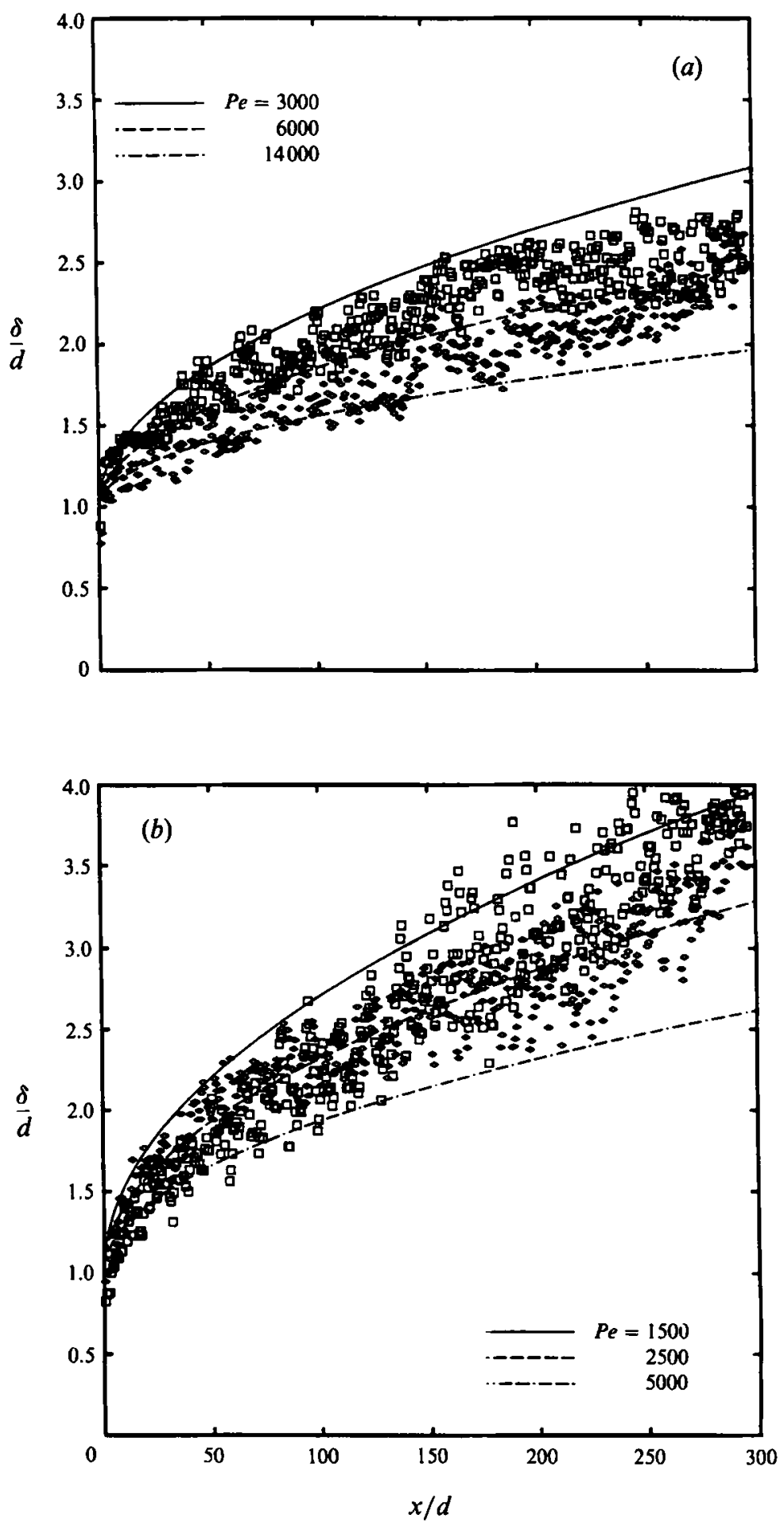

FIGURE 6. The growth of the mixing-layer thickness, $\delta / d$, as a function of downstream distance, $x / d$, for $(a)$ the wide channel with sawtooth walls, $\dot{m}=0.10 \mathrm{~kg} / \mathrm{s}(\diamond)$, and $\dot{m}=0.20 \mathrm{~kg} / \mathrm{s}(\square) ;(b)$ the narrow channel with sawtooth walls, $\dot{m}=0.074 \mathrm{~kg} / \mathrm{s}(\diamond)$, and $\dot{m}=0.14 \mathrm{~kg} / \mathrm{s}(\square)$. 

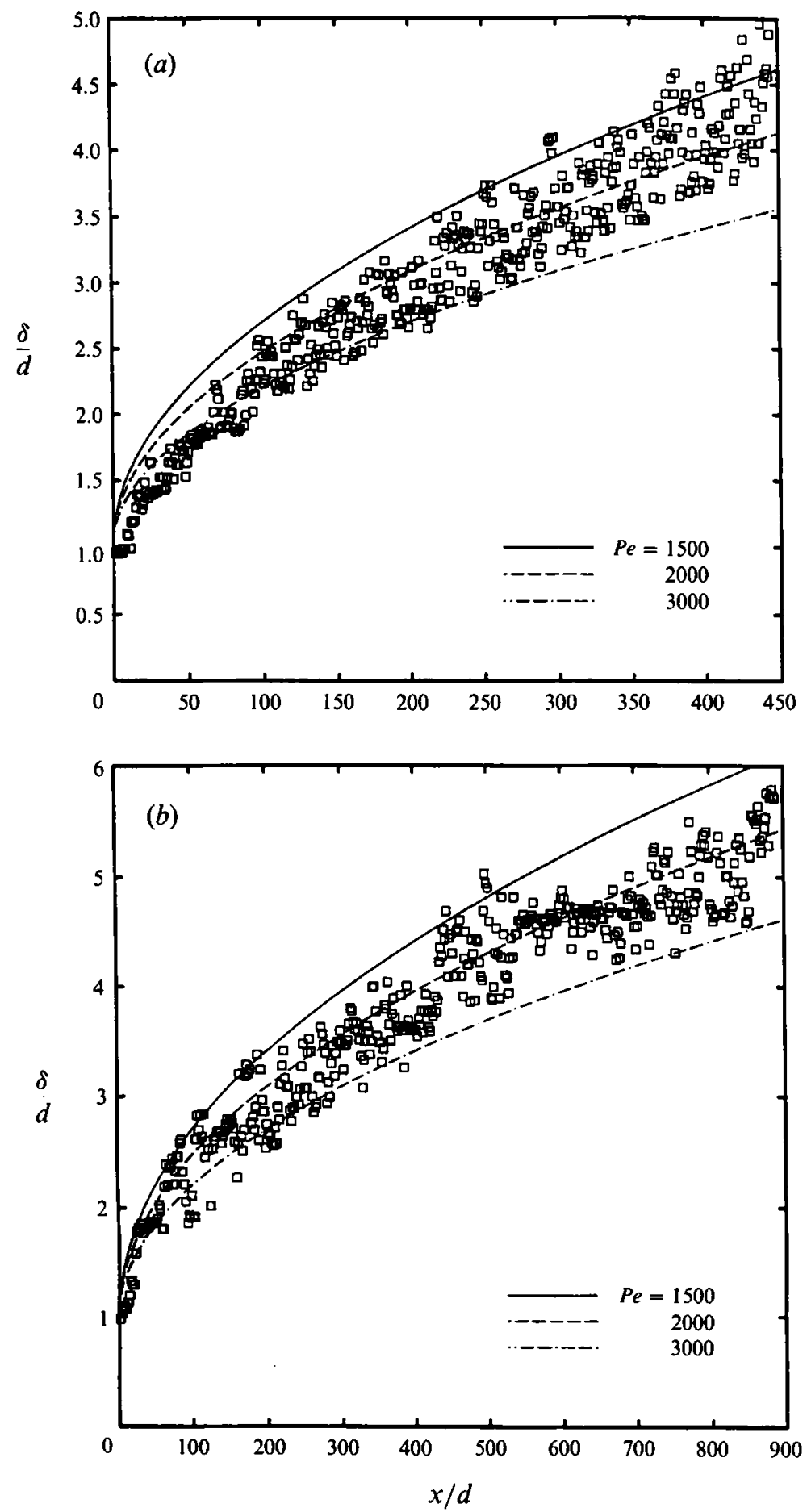

FIGURE 7 . The growth of the mixing-layer thickness, $\delta / d$, as a function of downstream distance, $x / d$, (a) using $2 \mathrm{~mm}$ beads with sawtooth walls, $\dot{m}=0.15 \mathrm{~kg} / \mathrm{s}$, and $2 H=2.54 \mathrm{~cm} ;(b)$ using $1 \mathrm{~mm}$ beads with sawtooth walls, $\dot{m}=0.077 \mathrm{~kg} / \mathrm{s}$, and $2 H=1.59 \mathrm{~cm}$ : $\square$, experiment. 
where the $m$ subscript is used to denote the average value within the mixing layer. Assuming that the diffusion process is not restricted by the channel boundaries, appropriate boundary conditions are that as $y \rightarrow \infty, C \rightarrow 1$, and as $y \rightarrow-\infty, C \rightarrow 0$; the initial conditions are that at $x=0$ and $y>0, C=1$, and at $x=0$ and $y<0, C=0$. Using these conditions, the governing equation is integrated, yielding the following concentration distribution:

$$
C(x, y)=\frac{1}{2} \pm \frac{1}{2} \operatorname{erf}\left(\frac{ \pm y}{2(x d)^{\frac{1}{2}}} P e^{\frac{1}{2}}\right),
$$

where the + is for $y>0$ and the - for $y<0$, and erf () indicates the error function. From this expression the mixing-layer thickness, $\delta / d$, is determined as

$$
\frac{\delta}{d}=6.60\left(\frac{x}{d} \frac{1}{P e}\right)^{\frac{1}{2}} .
$$

The analysis used to develop (4) assumes that the thickness of the mixing layer is zero at $x=0$. However, because of the thickness of the splitter plate in the experiments the experimental value of the initial mixing-layer thickness is probably closer to one particle diameter. As a result, the curves calculated using (4) are shifted by one particle diameter so that $\delta / d=1$ at $x / d=0$ as shown in figures $5-7$.

The lines of constant $P e$ are included for reference, and are drawn to bound the data. A comparison of the curves with the experimental measurements shows that $\delta / d$ does appear to increase with $(x / d)^{\frac{1}{2}}$. In addition, as mentioned in the introduction, the kinetic-theory studies by Savage (1992) and Hsiau \& Hunt (1992) demonstrate that the diffusion coefficient is proportional to the square-root of the granular temperature and the particle diameter. Since the granular temperature is not measured in the present experiment, it is impossible to apply directly this result. However, by assuming $\left\langle u^{\prime 2}\right\rangle^{\frac{1}{2}}$ is proportional to the square-root of the granular temperature, the diffusion coefficient $D_{\mathrm{m}}$ would depend on the product $d\left\langle u^{\prime 2}\right\rangle^{\frac{1}{2}}$, and the Péclet number follows as:

$$
P e=u_{\mathrm{m}} d / D_{\mathrm{m}} \propto u_{\mathrm{m}} /\left\langle u^{\prime 2}\right\rangle^{\frac{1}{2}} .
$$

This proportionality given by (5) suggests that as the ratio of the fluctuating velocity to the average velocity increases, the Péclet number decreases because of the increase in the diffusion coefficient; as a result, $\delta / d$ should increase. The roughened-wall and sawtooth-wall experiments indicate that for the higher flow rates, the velocity fluctuations increase as a percent of the local velocity, and the corresponding mixinglayer thicknesses are slightly larger. However, the dependence of the mixing-layer thickness on the longitudinal velocity fluctuations does not explain why $\delta / d$ is larger for the sawtooth walls than for the roughened walls when the profiles of the magnitude of the velocity fluctuations are similar for approximately equal flow rates and channel sizes. As stated previously, the curves are based on the assumption of a constant velocity and diffusion coefficient within the mixing layer. Although the measurements indicate that the local longitudinal velocity and the velocity fluctuations are relatively constant within the central region of the channel, visual observations show that the transverse particle motions vary within the flow. As a result, it is anticipated that the rate of particle diffusion does vary within the mixing layer.

Although the simple diffusion analysis does not incorporate all of the effects exhibited by the experiments, the lines suggest an appropriate scaling of the diffusion process with particle diameter. As indicated by (5), the Péclet number is independent of the particle diameter for the same magnitude of velocity fluctuation. By comparing 
the sawtooth-wall results in the channel of $2 H / d=12.7$ for the $2 \mathrm{~mm}$ beads with that for the $3 \mathrm{~mm}$ ones at the high flow rate (figures $6(b)$ and $7(a)$ ), the growth of the mixing layer for each flow roughly follows the curves corresponding to $P e$ between 1500 and 3000 . For the $1 \mathrm{~mm}$ beads, the velocity profiles were not measured and the ratio of the channel width to the particle diameter does not correspond exactly with that for the larger beads; however, the growth of the mixing-layer thickness appears to follow the same Péclet number curves as used for the larger beads.

\section{Conclusions}

The current experiments are designed to investigate the diffusion of particles that occurs when a granular material flow is sheared. In the experiments a range of flow conditions were investigated by varying the surface conditions of the sidewalls, the flow rates, the walls spacing and the particle sizes. The measured velocity profiles indicate a shear region near the wall of about 3-4 particles diameters in thickness, and a slip velocity that ranges from $25-60 \%$ of the average velocity, depending on the wall conditions. The longitudinal velocity fluctuations range from 10 to $16 \%$ of the average value, and increase with the overall flow rate. The diffusion measurements show that the mixing-layer thickness increases as the square-root of the downstream distance, and appears to depend on the magnitude of the fluctuating velocity as compared to the average velocity. The particle diffusion occurs within the central region of the flow in which the velocity measurements do not indicate any shearing of the material, though visual observations suggest some transverse movement of the particles. The experiments do not include the fluctuating velocity in the direction transverse to the flow. These measurements are the subject of future work and are important in defining the flow diffusion coefficient and in estimating the magnitude of particle mixing within a shear flow.

The authors would like to thank Professors Christopher Brennen and Rolf Sabersky for their helpful discussions and encouragement.

\section{REFERENCES}

AHN, H. 1989 Experimental and analytical investigations of granular materials: shear flow and convective heat transfer. PhD thesis, California Institute of Technology; Rep. E200.28.

AhN, H., Brennen, C. E. \& SABersky, R. H. 1991 Measurements of velocity, velocity fluctuation, density, and stresses in chute flows of granular materials. Trans. ASME E: J. Appl. Mech. 58, 792-803.

Bridgwater, J. 1980 Self-diffusion coefficients in deforming powders. Powder Tech. 25, 129-131.

BugGisch, H. \& LoffElmaNN, G. 1989 Theoretical and experimental investigations into local granulate mixing mechanisms. Chem. Engng Process. 26, 193-200.

CAMPBell, C. S. 1989 The stress tensor for simple shear flows of a granular material. J. Fluid Mech. 203, 449-473.

CAMPBell, C. S. 1990 Rapid granular flows. Ann. Rev. Fluid Mech. 22, 57-92.

Campbell, C. S. \& Brennen, C. E. 1985 Computer simulations of granular shear flows. J. Fluid Mech. 52, 167-188.

Chapman, S. \& Cowling, T. G. 1971 The Mathematical Theory of Non-uniform Gases, 3rd edn. Cambridge University Press.

Hsiau, S. S. \& HUNT, M. L. 1992 Kinetic theory analysis of flow-induced particle diffusion and thermal conduction in granular material flows. J. Heat Transfer (in press); also in General Papers in Heat Transfer, HTD 204 (ed. R. Jensen et al.), pp. 41-48. ASME, New York. 
Hunt, M. L. \& Hsiau, S. S. 1992 Experimental measurements of particle diffusion and velocity profiles in a granular-flow mixing layer. In Advances in Micromechanics of Granular Materials (ed. H. H. Shen et al.), pp. 141-150. Elsevier.

Hwang, C. L. \& HoGg, R. 1980 Diffusive mixing in flowing powders. Powder Tech. 26, 93-101.

Jenkins, J. T. \& Savage, S. B. 1983 A theory for the rapid flow of identical, smooth, nearly elastic, particles. J. Fluid Mech. 130, 187-202.

Johnson, P. C., NotT, P. \& JACKson, R. 1990 Frictional-collisional equations of motion for particulate flows and their application to chutes. J. Fluid Mech. 210, 501-535.

KenNaRd, E. H. 1938 Kinetic Theory of Gases. McGraw-Hill.

Lun, C. K. K., Savage, S. B., Jeffrey, D. J. \& Chepurniy, N. 1984 Kinetic theories for granular flow: inelastic particles in Couette flow and slightly inelastic particles in a general flowfield. $J$. Fluid Mech. 140, 223-256.

SAVAGE, S. B. 1979 Gravity flow of cohesionless granular materials in chutes and channels. $J$. Fluid Mech. 92, 53-96.

SAVAGE, S. B. 1992 Disorder, diffusion and structure formation in granular flows. In Disorder and Granular Media (ed. D. Bideau). Elsevier.

SAVAGE, S. B. \& JefrReY, D. J. 1981 The stress tensor in a granular flow at high shear rates. J. Fluid Mech. 110, 255-272.

WALTON, O. R. \& BRAUN, R. L. 1986 Viscosity, granular-temperature, and stress calculations for shearing assemblies of inelastic, frictional disks. J. Rheol. 30, 949-980.

WANG, D. G. \& CAMPBELL, C. S. 1992 Reynolds' analogy for a shearing granular material. J. Fluid Mech. 244, 527-546. 